J Craniofac Surg. 2009 September ; 20(Suppl 2): 1652-1654. doi:10.1097/SCS.0b013e3181b2d3f3.

\title{
Genetics and Human Malformations
}

\author{
Gabriele Mues, MD ${ }^{1}$, Hitesh Kapadia, DDS, $\mathrm{PhD}^{2}$, Ying Wang, DDS, $\mathrm{PhD}^{1}$, and Rena N. \\ D'Souza, DDS, PhD' \\ ${ }^{1}$ Department of Biomedical Sciences, Texas A\&M University Health Science Center Baylor College \\ of Dentistry, Dallas, TX 75246, USA \\ ${ }^{2}$ New York University Medical Center, Institute of Reconstructive Plastic Surgery, 560 First Avenue, \\ New York, NY 10016
}

\begin{abstract}
Genetics gains more and more importance in all areas of health care including craniofacial surgery and dentistry. This does not mean that every patient will benefit from genetic advances but for many health problems we will see progress in explaining disease pathogenesis, establishing diagnosis, guiding therapy, predicting prognosis and achieving prevention. In this report we briefly review the roles of the PAX9, MSX1, AXIN2 and EDA genes in the causation of congenital tooth agenesis and the promise of molecular genetic research for the improvement of patient care.
\end{abstract}

\section{Keywords}

benefits of genetics; craniofacial medicine; tooth agenesis genes

Every person harbors in their genome many small and seemingly random variations which constitute the major basis for our individual differences. In most individuals, these variants do not adversely affect the health and phenotypic appearance of their bearer. In others however, a single gene variant or a combination of several gene variations may lead to effects that exceed our norm for structural and/or functional variation.

Craniofacial structures, including teeth are often affected by malformations. This is not surprising when one considers the complexity of gene interactions that must be precisely coordinated in space and time and at an appropriate dosage to develop a complicated structure like the human head. Genetic variants that are sufficient for the formation of other organs may not be able to function properly during craniofacial development, with or without additional environmental cofactors such as physical constraint in utero, nutritional deficiencies, pharmacological agents, toxins, infections and radiation. Alternatively, the same gene variant (s) may also cause disturbances elsewhere in the body plan. The craniofacial malformations, however, are usually perceived as the most unsettling ones.

Due to advancements made in craniofacial surgery, much of the morbidity and mortality in infants born with malformations can be alleviated. In light of this, one may be tempted to consider the problem as solved without the need to identify the genetic basis. However, knowing the underlying gene(s) does more than just satisfy scientific curiosity. In both medicine and more recently in dentistry, genetics has a central role in explaining disease pathogenesis, establishing diagnosis, guiding therapy and predicting prognosis. Benefits for

Address correspondence to: R. N. D'Souza, Department of Biomedical Sciences, Texas A\&M University Health Science Center Baylor College of Dentistry, 3302 Gaston Avenue, Dallas, TX 75246, USA. Phone: 214828 8375, Fax: 214874 4538, rd'souza@ bcd.tamhsc.edu. 
patients include early diagnosis and intervention, genetic counseling to help the family with reproductive decisions and more targeted treatments. Eventually, even causative treatments or cure may be achievable. In addition, knowledge about the underlying genetic and molecular deficits can lead to the creation of better animal models for studying the disease process and for the advancement of craniofacial surgical procedures and outcome studies.

The foundation for gene discovery is often built by the clinician through keen clinical observation, detailed phenotype description and the stratification of these findings. The craniofacial surgeon is many times the first in line to contribute to this process by identifying and recruiting patients and their families for participation in genetic studies. Mutations in fibroblast growth factor receptor genes(FGFRs)as causes for several syndromic conditions such as Crouzon, Apert and Kallmann (1-3) have been uncovered this way. Very often, patients and their families who consent to participate in a study that attempts to find the root of their medical problem derive a positive sense of purpose and usefulness from their commitment. Another avenue to gene discovery starts in the laboratory with experimental mouse models. Most genes in mice can be manipulated at will, the ensuing phenotype studied and correlated with findings in human syndromes. This approach is very useful for the identification of candidate genes, i.e. genes that are most likely involved in a certain disease process or syndrome.

\section{Genes in human tooth agenesis}

The formation of teeth is one example of the intricate cooperation of different genetic pathways that lead to the morphogenesis of human facial features. Animal studies have shown that tooth development requires a sequential array of epithelial-mesenchymal interactions involving many signaling molecules like growth factors and their receptors, transcription factors and further modifier proteins. Interestingly, there is remarkable conservation of many of these pathways, as they are also employed in the shaping of other craniofacial structures (4).

Human tooth agenesis can present either as an isolated trait or as part of a syndrome. The isolated, non-syndromic form is quite common even if missing wisdom teeth are disregarded. Most often it affects just one or two upper lateral incisors or premolars. There are, however, many families or individuals with a history of severe tooth agenesis. Interestingly, different patterns of missing teeth such as mostly posterior, more anterior, mostly incisor or totally mixed are also observed.

Genetic and molecular studies in mice have shown that the paired box protein Pax9 and the homeobox protein Msx1 are mesenchymally-expressed transcription factors which are selectively activated in the early tooth bud and are required for the maintenance of Bmp4 expression (bone morphogenetic protein 4). Bmp4 is a signaling molecule required for the formation of an epithelial signaling center, the enamel knot and directs progression to the next stage of odontogenesis. Mice in which Pax9 and/or Msx 1 are completely missing show an arrest of tooth development at the bud stage (5).

Over the past decade, we and others have analyzed families with tooth agenesis for genetic variations in these genes and discovered that some of the families with a more anterior pattern of tooth agenesis had MSX1 mutations while others with a posterior pattern showed changes in the PAX9 gene (Figure 1A). The extension of our investigations to functional studies demonstrated that Pax9 but not Msx1 was able to activate both Bmp4 and Msx1 expression. However, the addition of Msx1 could amplify Pax9-mediated Bmp4 and Msx1 activation considerably (Figure 1C). In contrast, the Pax9 mutants that had been identified in the tooth agenesis families were not able to activate Bmp4 and Msx 1 expression(6). We have also shown that the mechanism of this loss of function is a deficit in binding of the Pax9 paired domain to the promoter DNA sequences of the two target genes(7). 
A third gene, $A X I N 2$, has been implicated in the mixed form of tooth agenesis after it was found by Lammi et al. (8) that certain AXIN2 mutations cause tooth agenesis and predispose to colon cancer in one large family and in a sporadic case. AXIN2 is a regulator in the Wnt signaling pathway, which is ubiquitously utilized throughout development and also includes the APC (adenomatous polyposis coli) gene product. Although the AXIN2 gene is expressed in dental mesenchyme along with many other genes, it was not anticipated to be a "tooth agenesis" gene because of its more widespread expression. Only a family study with linkage analysis could reveal its importance for odontogenesis.

The fourth tooth agenesis gene $(9,10)$ turned out to be a gene which is well-known by geneticists for its role in the syndrome hypohidrotic ectodermal dysplasia (HED). An isolated form of tooth agenesis was not expected to be associated with $E D A$ mutations and we were quite surprised to find an $E D A$ mutation in our largest tooth agenesis family (Figure 1B). Once $E D A$ had been identified as a non-syndromic tooth agenesis gene, more and more families with $\mathrm{X}$-linked isolated tooth agenesis were shown to have an $E D A$ gene mutation. It is predominantly responsible for incisor agenesis patterns. EDA belongs to the family of tumor necrosis factorlike proteins which are signaling molecules and as such, released from their cells of origin. After binding to their specific target receptors on recipient cells, they activate the NFKB pathway, which consists of a complex assortment of intracellular proteins. The EDA receptor EDAR and two of the intracellular accessory proteins called EDARADD and NEMO can also cause ectodermal dysplasia-like syndromes if mutated, though NEMO mutations are associated with the phenotypically distinct syndromes incontinentia pigmenti in females and ectodermal dysplasia with immunodeficiency in surviving males.

In collaboration with a Swiss research group we sought to determine the functional differences between EDA mutations that cause full-blown HED and those that are found in isolated tooth agenesis. We were able to show that the former lose all signaling capability while the latter retain residual function (manuscript in preparation). Thus we conclude that tooth development requires a higher dosage of EDA signaling than hair and gland development. The detailed knowledge about the EDA protein and its function as an extracellular signaling molecule led one group of researchers to try a remarkable experiment $(11,12)$ : They attempted to alleviate the ectodermal dysplasia phenotype in HED-affected mice and dogs by administration of recombinant EDA protein. The experiment was successful!

To date, four genes have been identified as causing tooth agenesis. There are, however, many patients with hypodontia who do not have a mutation in any of these genes. Thus we continue our search, assisted by ever advancing DNA sequencing and data-processing technologies and the human genome data base.

\section{Acknowledgments}

The study was supported by funds from NIH U24-PEI6472 to RDS

\section{References}

1. Wilkie AOM, Slaney SF, Oldridge M, et al. Apert syndrome results from localized mutations of FGFR2 and is allelic with Crouzon syndrome. Nature Genet 1995;9:165-172. [PubMed: 7719344]

2. Reardon W, Winter RM, Rutland P, et al. Mutations in the fibroblast growth factor receptor 2 gene cause Crouzon syndrome. Nature Genet 1994;8:98-103. [PubMed: 7987400]

3. Dode C, Levilliers J, Dupont JM, et al. Loss-of-function mutations in FGFR1 cause autosomal dominant Kallmann syndrome. Nature Genet 2003;33:463-465. [PubMed: 12627230]

4. Thesleff I. Epithelial-mesenchymal signaling regulating tooth morphogenesis. J Cell Sci 2003;116:1647-1648. [PubMed: 12665545] 
5. Peters HA, Neubuser, et al. Pax genes and organogenesis: Pax9 meets tooth development. Eur J Oral Sci 1998;106 (Suppl 1):38-43. [PubMed: 9541201]

6. Ogawa T, Kapadia H, Feng JQ, et al. Functional consequences of interactions between Pax9 and Msx1 genes in normal and abnormal tooth development. J Biol Chem 2006;281:18363-18369. [PubMed: 16651263]

7. Wang Y, Groppe JC, Wu J, et al. Pathogenic mechanisms of tooth agenesis linked to paired domain mutations in human PAX9. 2009 submitted.

8. Lammi L, Arte S, Somer M, et al. Mutations in AXIN2 cause familial tooth agenesis and predispose to colorectal cancer. Am J Hum Genet 2004;74:1043-1050. [PubMed: 15042511]

9. Tao R, Jin B, Guo SZ, et al. A novel missense mutation of the EDA gene in a Mongolian family with congenital hypodontia. J Hum Genet 2006;51:498-502. [PubMed: 16583127]

10. Tarpey P, Pemberton TJ, Stockton DW, et al. A novel Gln358Glu mutation in ectodysplasin with Xlinked dominant incisor hypodontia. Am J Med Genet 2007;143A:390-394. [PubMed: 17256800]

11. Gaide O, Schneider P. Permanent correction of an inherited ectodermal dysplasia with recombinant DNA. Nature Medicine 2003;9:614-618.

12. Casal ML, Lewis JR, Mauldin EA, et al. Significant correction of disease after postnatal administration of recombinant ectodysplasin A in canine X-linked ectodermal dysplasia. Am J Hum Genet 2007;81:1050-1060. [PubMed: 17924345] 
A

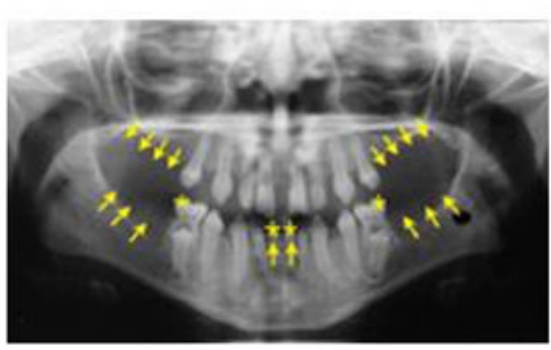

B

B

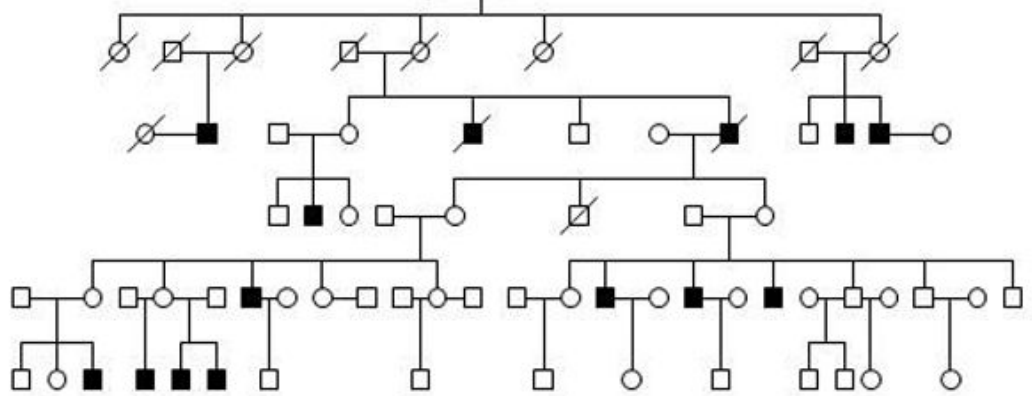

C

$\mathrm{Pax9}$ (ng) Msx1(ng) p2.4Bmp4-Luc

Figure 1.

A Panorex of a patient with non-syndromic posterior tooth agenesis caused by a PAX9 mutation. B Pedigree of a family with non-syndromic incisor agenesis caused by the EDA gene. In this X-linked condition only males are affected (black squares). C Activation of a Bmp4 promoter construct by Pax9, Msx1 and a combination of Pax9 and Msx1. 